\title{
SINGULAR HOMOLOGY AS A DERIVED FUNCTOR(1)
}

BY

\author{
G. S. RINEHART ${ }^{*}$
}

\begin{abstract}
A general theory of relative derived tunctors is applied to the category of topological spaces to obtain singular homology and cohomology, verify the Eilenberg-Streenrod axioms, and show that singular and simplicial theory agree.
\end{abstract}

Introduction. In [2] satellites relative to a projective class are defined for functors with nonadditive domain. The applications there are algebraic. The domain categories are groups, algebras, etc., and the usual cohomology groups are obtained as satellites and their properties (mainly those dealing with extensions) are derived in a unified and cochain-free manner. Our object here is to treat singular homology and cohomology in the same fashion.

In $\$ 1$ the relevant formal machinery of [2] is a summarized. (It appears there as the first four pages of the second section.) This is done both for the sake of completeness and because the results have been subjected to mild simplification and generalization to make them suitable for our present purposes. (Anyone who attempts to read further in [2] should be warned that there are many small errors, only some of which can be attributed to the fact that the paper was published without the author having seen the galley proofs. In particular, B3 must be omitted from the list in the first section (it is equivalent to closure), and, in $\mathrm{B} 4, A \rightarrow B$ should be taken to be in the projective class, and " $\Leftrightarrow$ " should be replaced by " $\Leftarrow "$. Also, in Proposition 2.20, $F$ must be as sumed right exact.) In $\$ 2$ of the present paper, the aforementioned formal machinery is applied to the category of topological spaces to obtain singular homology and produce short proofs of the homotopy axiom and the Eilenberg-Zilber Theorem. In order to obtain the Mayer-Vietoris sequences equivalent to the excision axiom, it is neces sary to pass to the category of formal inverse limits of topological spaces, and once this has been done it is easy to show that singular and simplicial theory agree on simplicial complexes. This is done in $\$ 3$.

Except in the case of the Eilenberg-Zilber Theorem, our results are given

Received by the editors September 24, 1971.

AMS (MOS) subject classifications (1970). Primary 18G10, 55J99; Secondary 55B 10.

Key words and phrases. Singular homology as a derived functor, Eilenberg-Steenrod axioms, comparison of singular and simplicial homology.

(1) This research was supported in part by N.S.F. contract GP 9395.

${ }^{*}$ Deceased November 2, 1972. All correspondence concerning this paper should be addressed to Alex Rosenberg, Department of Mathematics, Cornell University, Ithaca, New York 14850. 
without specifying the range category. The appropriate theorems for homology and cohomology are obtained by taking the range to be respectively the category of abelian groups or its dual.

The theory of satellites used here is a very simple special case of Grothendieck cohomology [0] in which the covers all consist of single morphisms. In fact, it is the intersection of this theory with that used by André (Springer Lecture Notes in Math., vol. 32), because we assume that there are enough projectives = models, so that a right exact functor $=$ sheaf is determined by its behavior thereon. It also agrees with cotriple cohomology. See [2, \$2 and Remark 5.6]. Barr-Beck have indicated how singular homology can be obtained via the latter (Springer Lecture Notes, vol. 80), so that the fact that it arises in this setting is not new.

1. Satellite functors. Let $\mathbf{C}$ be a category with fiber products, $\mathbf{E}$ a class of morphisms in $\mathbf{C}$ closed under composition, and let $\mathbf{P}$ be a set of objects in $\mathbf{C}$ (called the projectives). (The assumption that $\mathbf{P}$ is a set will turn out to be harmless. See Remark 2.3.) We require that, if $f \in \mathbf{E}, \mathbf{C}(P, f)$ be surjective $f$ or every $P \in \mathbf{P}$; and, for every object $X$ in $\mathbf{C}$, there be a $P \rightarrow X \in \mathbf{E}$ with $P \in \mathbf{P}$. Such a triple (C, P, E) will be called a base.

Throughout the sequel, $\mathbf{A}$ will denote an abelian category with exact infinite coproducts and enough projectives (in the usual sense). A will typically be the category of abelian groups or its dual.

Definition 1.1. A functor $F: \mathrm{C} \rightarrow \mathrm{A}$ respects a morphism $X \rightarrow Y$ in case the sequence $F\left(X \times_{Y} X\right) \rightarrow F(X) \rightarrow F(Y) \rightarrow 0$ is exact, where the first morphism is the difference of the morphisms corresponding to the canonical projections. $F$ is right exact in case it respects every morphism in $\mathbf{E}$. Denote the category of all functors from $\mathbf{C}$ to $\mathbf{A}$ by $\mathbf{A}^{\mathrm{C}}$, and the full subcategory of all right exact functors by $\mathbf{R}(\mathbf{C}, \mathbf{A}, \mathbf{E})$. (We will suppress as much of the data in parentheses as the context allows.) Denote by $A^{P}$ the category of all functors to $A$ from the full subcategory of $\mathbf{C}$ with objects $\mathbf{P}$.

Remark 1.2. Consider a composition of morphisms $X \rightarrow Y \rightarrow Z$. If $F$ respects $X \rightarrow Z$, and if $F(X) \rightarrow F(Y)$ is an epimorphism, then $F$ respects $Y \rightarrow Z$. Again, if $F$ respects $X \rightarrow Y$ and $Y \rightarrow Z$, and if $F\left(X \times_{Z} X\right) \rightarrow F\left(Y \times_{Z} Y\right)$ is an epimorphism, then $F$ respects $X \rightarrow Z$.

Proof. Consider the diagram

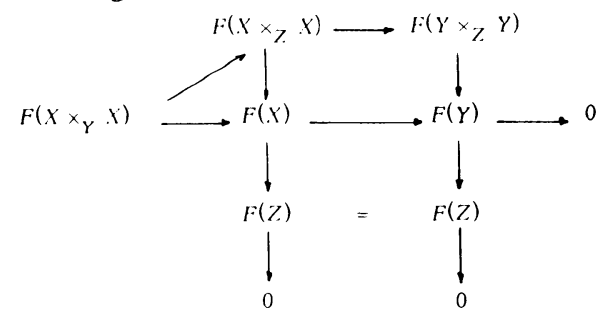


Corollary. If $F$ respects all morphisms $P \rightarrow X \in \mathbf{E}, P \in \mathbf{P}$, then $F$ is right exact.

In the sequel, we shall also use the terms "projective" and "right exact" in their usual sense in the context of abelian categories. This corresponds to taking $\mathbf{E}$ to be the class of all epimorphisms.

Specifying a right exact functor on projectives determines it completely. This fact is embodied in [2, Proposition 2.4], where a functor $\mathbf{A}^{\mathbf{P}} \rightarrow \mathbf{R}$, denoted $F \mapsto F^{\#}$, is constructed. (The last part of the proof of this proposition is unnecessary: That $G^{\#}$ is right exact is immediate from the above corollary.) If $P \in \mathbf{P}$, then $F^{\sharp}(P)=F(P)$. If $G$ is the restriction of $F \in|\mathbf{R}(\mathbf{E})|$, then $G^{\#}=F$. The restriction $R \rightarrow A^{P}$ is thus shown to be an equivalence of categories, so that $R$ is abelian with enough projectives. Moreover, the composition $A^{C} \rightarrow A^{P} \rightarrow R$ (also denoted $F \rightarrow F^{\#}$ ) is a right adjoint to the natural inclusion, so that, in particular, the latter is right exact. $S_{n}: \mathbf{R} \rightarrow \mathbf{A}^{\mathrm{C}}$ is defined to be the $n$th left derived functor of the inclusion functor. Then, since, by the above category equivalence, exactness in $\mathbf{R}$ is exactness at projectives,

Proposition 1.3. $S_{n} F(P)=0$ for $n \geq 1, P \in \mathbf{P}$.

Given a morphism $X \rightarrow Y$, the corresponding $\check{C} e c b$ bomology groups of $F$, $H_{*}(X \rightarrow Y, F)$, are the homology groups of the complex $\cdots \rightarrow F\left(X \times_{Y} X \times_{Y} X\right) \rightarrow$ $F\left(X \times_{Y} X\right) \rightarrow F(X)$, the boundary maps being the alternating sum of the canonical projections [2, Definition 2.2], so that $F$ respects $X \rightarrow Y$ if and only if $H_{0}(X \rightarrow Y, F) \rightarrow F(Y)$ is an isomorphism. $F$ is flask at $X \rightarrow Y$ in case $F$ respects $X \rightarrow Y$ and $H_{n}(X \rightarrow Y, F)=0$ whenever $n \geq 1$. If $\mathbf{M}$ is a class of morphisms, we will say $F$ is $\mathbf{M}$-flask if it is flask at every morphism in M. " $F$ is flask" means $F$ is E-flask. (Note that we here require flask functors to be right exact. This is not done in [2].) If $F$ is flask, then $S_{n} F=0$ whenever $n \geq 1$ [2, Proposition 2.12]. Hence, in general, $S_{n} F$ can be computed using a resolution of $F$ composed of flasks. Projectives in $\mathbf{R}$ are flask [2, Corollary 2.11].

Definition 1.4. Let $\left(\mathbf{C}_{i}, \mathbf{P}_{i}, \mathbf{E}_{i}\right)$ be bases, $i=1,2$. A morphism of bases is a functor $\Lambda: C_{1} \rightarrow C_{2}$ which preserves fiber products and carries $E_{1}$ to $E_{2}$.

Note that the morphism $A^{C_{2}} \rightarrow A^{C_{1}}$ defined by composition with $\Lambda_{\text {restricts }}$ to a morphism from $R_{2}=\mathbf{R}\left(C_{2}\right)$ to $R_{1}$. Note also that composition with $\Lambda$ preserves flasks. From this we conclude the key Comparison Theorem:

Theorem 1.5. Let $\Lambda: \mathrm{C}_{1} \rightarrow \mathrm{C}_{2}$ be a morphism of bases. Let $F$ be $\mathrm{E}_{2}$-right exact, and suppose that, for every $P \in \mathbf{P}_{1}, S_{n} F(\Lambda(P))=0$ whenever $n \geq 1$. Then $\left(S_{n} F\right) \Lambda=S_{n}(F \Lambda)$.

Proof. Consider a projective resolution of $F$ in $\mathbf{R}_{2}$. Upon composition with $\Lambda$, we obtain a complex over $F \Lambda$ in $\mathbf{R}_{1}$ composed of flasks. At $P \in \mathbf{P}_{1}$, this complex 
has $n$th homology group $S_{n} F(\Lambda(P))$. Hence, since exactness in $\mathbf{R}_{2}$ is exactness at projectives, it is a resolution of $F \Lambda$ in $\mathbf{R}_{1}$. We conclude that $S_{n}(F \Lambda)$ is the value at $F$ of the $n$th derived functor of the composition $\mathbf{R}_{2} \rightarrow \mathbf{R}_{1} \rightarrow \mathbf{A}^{\mathrm{C}_{1}}$, where the first functor is composition with $\Lambda$. But this can also be written $R_{2} \rightarrow A^{C_{2}} \rightarrow A^{C_{1}}$, where the second functor is composition with $\Lambda$, which is always exact on the category of all functors. This gives the desired result.

\section{Singular homology and formal properties.}

Definition 2.1. Let $\mathrm{C}^{2}$ be the category whose objects are the morphisms of $\mathbf{C}$, and whose morphisms are commutative squares in $\mathbf{C}$. We suppose that $\mathbf{C}$ has an initial object, denoted 0 , that $0 \in \mathbf{P}$, and that, for all $P, P^{\prime} \in \mathbf{P}$, there is a coproduct $P+P^{\prime} \in \mathbf{P}$. Write an object $U \rightarrow X$ in $\mathbf{C}^{2}$ as $(X, U)$. Let $\mathbf{P}^{2}$ consist of all objects $\left(P+P^{\prime}, P\right)$ (canonical map), where $P, P^{\prime} \in \mathbf{P}$. Let $\mathbf{E}^{2}$ be all morphisms $(X, U) \rightarrow(Y, V)$ in $\mathbf{C}^{2}$ with $U \rightarrow V$ and $X \rightarrow Y$ in $\mathbf{E}$. Then $\left(\mathbf{C}^{2}, \mathbf{P}^{2}, \mathbf{E}^{2}\right)$ is a base. Given an E-right exact $F: \mathbf{C} \rightarrow \mathbf{A}$ with $F(0)=0$, extend $F$ to $\mathbf{C}^{2}$ by defining $F(X, U)=$ cokernel $F(U) \rightarrow F(X)$. Then, since $X \mapsto(X, 0)$ is a morphism of bases which preserves projectives, $S_{n} F(X)=S_{n} F(X, 0)$ by the Comparison Theorem. Define $\Gamma_{0}: \mathbf{C}^{2} \rightarrow \mathrm{C}$ by $\Gamma_{0}(X, U)=U$. Similarly, define $\Gamma_{1}(X, U)=X$. These are projective preserving morphisms of bases. Suppose that $F(P) \rightarrow$ $F\left(P+P^{\prime}\right)$ is always injective. Then the sequence $0 \rightarrow F \Gamma_{0} \rightarrow F \Gamma_{1} \rightarrow F \rightarrow 0$ is exact in $\mathbf{R}\left(\mathbf{C}^{2}\right)$. Since $S_{n}\left(F \Gamma_{i}\right)=\left(S_{n} F\right) \Gamma_{i}$, we obtain a natural exact sequence

$$
\cdots \rightarrow S_{n+1} F(X, U) \rightarrow S_{n} F(U) \rightarrow S_{n} F(X) \rightarrow S_{n} F(X, U) \rightarrow \cdots
$$

for every $(X, U) \in\left|\mathrm{C}^{2}\right|$.

Remark 2.3. We may now drop the assumption that $\mathbf{P}$ is a set, since $S_{n} F$ can be defined "locally" on small full subcategories chosen so that the inclusion functor is a projective preserving morphism of bases (cf. [2, Remark 2.16]).

Remark 2.4. Suppose that $\mathbf{P}_{0}$ is a set of objects of a category $\mathbf{C}$, and that $\mathbf{C}$ has fiber products and contains arbitrary coproducts of objects from $\mathbf{P}_{0}$. Let $\mathbf{P}$ be all such coproducts. Let $\mathbf{E}$ be all morphisms $f$ in $\mathbf{C}$ such that $\mathbf{C}(P, f)$ is surjective for every $P \in \mathbf{P}_{0}$. (One writes $\mathbf{P}_{0} \Rightarrow \mathbf{E}$.) Given $X$, let $P$ be the coproduct, for each $C \in \mathbf{P}_{0}$, of the coproduct of a copy of $C$ for each morphism from $C$ to $X$. Then there is a canonical $P \rightarrow X \in \mathbf{E}$. Hence $(\mathbf{C}, \mathbf{P}, \mathbf{E})$ is a base.

Definition 2.5. Let $T$ be the category of topological spaces and continuous maps, and let $\mathbf{P}_{0}$ be the set of cubes $I^{n}, n=0,1,2, \ldots$. Form $\mathbf{P}, \mathbf{E}$ as above. Given $A \in|\mathbf{A}|$, we will define an associated right exact functor, still denoted $A$. Let $A$ be the constant functor with value $A$ on objects in $\mathbf{P}_{0}$, and extend it to objects in $\mathbf{P}$ by decreeing that it preserve coproducts. By the category isomorphism $\mathbf{R} \cong \mathbf{A}^{\mathbf{P}}$ of $\$ 1$, this determines $A$. If $\mathbf{A}$ is abelian groups, write $H_{n}(X, A)=$ $S_{n} A(X)$, and, more generally, $H_{n}(X, U ; A)=S_{n} A(X, U)$ (Definition 2.1). If $\mathbf{A}$ is the 
dual of abelian groups, write $H^{n}(X, U ; A)=S_{n} A(X, U)$, considered as a functor to abelian groups contravariant in $(X, U)$. (More generally, we can replace the category of abelian groups by a category of modules.) We will continue to use " + " for the coproduct (disjoint union) in T.

Computation via the singular complex.

Proposition 2.6. Suppose that the map from $U$ to $X$ is injective. Let $G_{n}(X, U)$ be the coproduct in $\mathbf{A}$ of one copy of $A$ for each map from $I^{n}$ to $X$ which cannot be factored continuously through U. Use the usual boundary operator (choosing bomeomorphisms $\Delta_{n} \cong I^{n}$ ) for singular bomology to form the complex $G(X, U)$. Then the nth bomology group of this complex is $S_{n} A(X, U)$.

Proof. Consider evaluation at $\left(I^{n}, I^{n}\right)$ as a functor from $\mathrm{A}^{\mathrm{T}^{2}}$ to A. Let $G_{n}^{\prime \prime}$ be the image of $A$ under the left adjoint to this functor: $G_{n}^{\prime \prime}(X, U)$ is the coproduct of one copy of $A$ for each map from $\left(I^{n}, I^{n}\right)$ to $(X, U)$; i.e., for each map from $I^{n}$ to $U$. Define $G_{n}^{\prime}$ similarly, using $I^{n}$ in place of $\left(I^{n}, I^{n}\right): G_{n}^{\prime}(X, U)$ is the coproduct of one copy of $A$ for each map from $I^{n}$ to $X$. We may replace $\mathrm{T}^{2}$ by the full subcategory of all pairs with injective map, since the inclusion functor is a projective preserving morphism of bases. Then we have an exact sequence $0 \rightarrow G_{n}^{\prime \prime} \rightarrow$ $G_{n}^{\prime} \rightarrow G_{n} \rightarrow 0$ in $\mathrm{A}^{\mathrm{T}^{2}}$. Moreover, $G_{n}^{\prime \prime}$ and $G_{n}^{\prime}$ are flask [2, Proposition 2.10]. Hence $G_{n}$ is right exact, and, from the long exact sequence $\cdots \rightarrow S_{m} G_{n}^{\prime}(X, U) \rightarrow$ $S_{m} G_{n}(X, U) \rightarrow S_{m-1} G_{n}^{\prime \prime}(X, U) \rightarrow S_{m-1} G_{n}^{\prime}(X, U) \rightarrow \ldots$ we conclude that $S_{m} G_{n}(X, U)=0$ for $m \geq 1$. Since exactness in $\mathbf{R}\left(\mathbf{T}^{2}\right)$ is exactness at each $\left(P, P^{\prime}\right) \in \mathbf{P}^{2}$, and since the homology of $G\left(P, P^{\prime}\right)$ is the singular homology of a pair of spaces each of which is a disjoint union of cubes, we conclude that the complex of functors $G$ is a resolution in $\mathbf{R}\left(\mathrm{T}^{2}\right)$ of the functor $A$. This gives the desired result.

We now proceed to verify the formal properties of singular homology and cohomology. We already have the dimension axiom (Proposition 1.3) and the exact sequence for an arbitrary map (2.2).

Homotopy. We will show that the projection map induces an isomorphism from $S_{n} A(X \times I, U \times I)$ to $S_{n} A(X, U)$. It then follows that the two maps in the reverse direction given by $x \mapsto(x, 0)$ and $x \mapsto(x, 1)$ define the same map on homology; namely, the inverse to the one induced by projection. By (2.2) and the Five Lemma, it suffices to show that $S_{n} A(X \times I) \rightarrow S_{n} A(X)$ is always an isomorphism. Define $\Lambda: \mathrm{T} \rightarrow \mathrm{T}$ by $\Lambda(X)=X \times I$. Then $\Lambda$ is a projective preserving morphism of bases. The identification given by the Comparison Theorem is clearly natural in $\Lambda$, so that we have a commutative diagram

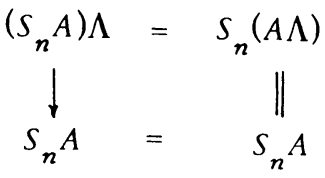


where the vertical maps are induced by the natural transformation from $\Lambda$ to the identity defined by projection. This is the desired result.

The Eilenberg-Zilber Theorem.

Proposition 2.7. Let $(\mathbf{C}, \mathbf{P}, \mathbf{E})$ be any base. Suppose $D: \mathbf{A}_{1} \rightarrow \mathbf{A}_{2}$ is a right exact functor between abelian categories which preserves coproducts. Let $F \in$ $\mathbf{R}\left(\mathbf{C}, \mathbf{A}_{1}\right)$ and suppose that $F(P)$ is projective when $P \in \mathbf{P}$. Let $G=\cdots \rightarrow G_{n+1}$ $\rightarrow G_{n} \rightarrow \cdots$ be a projective resolution of $F$. Then $D G$ is a flask resolution of $D F \in \mathbf{R}\left(\mathbf{C}, \mathbf{A}_{2}\right)$, so that $S_{n}(D F)(X)=H_{n}(D G(X))$, the nth bomology group of $D G(X)$.

Proof. Every projective in $\mathbf{R}\left(\mathbf{C}, \mathbf{A}_{1}\right)$ is a direct summand of a coproduct of functors $\langle P, A\rangle, P \in \mathbf{P}, A$ a projective in $\mathbf{A}_{1}$, where $\langle P,-\rangle$ is the left adjoint of evaluation at $P:\langle P, A\rangle(X)$ is the coproduct of one copy of $A$ for each morphism in $\mathbf{C}(P, X)$ [2, Corollary 2.5 and Proposition 2.10]. $D\langle P, A\rangle=$ $\langle P, D(A)\rangle$, and the latter is flask [2, Corollary 2.11]. Hence $D G$ consists of flasks. By the above, projectives of $R\left(C, A_{1}\right)$ take values in projectives of $A_{1}$. Hence $G(P)$ is a projective resolution of $F(P)$ when $P \in \mathbf{P}$, so that $H_{n}(D G(P))=$ $L_{n} D(F(P))=0, n \geq 1$. The conclusion follows.

Corollary. Let $\mathbf{A}=R$-modules, $R$ commutative, and let $A \in \mathbf{A}$. Let $G$ be a projective resolution of $R \in \mathbf{R}(\mathbf{T}, \mathbf{A})$. Then

$$
H_{n}(X, A)=H_{n}\left(G(X) \otimes_{R} A\right), \quad H^{n}(X, A)=H^{n}\left(\operatorname{Hom}_{R}(G(X), A)\right) .
$$

Theorem 2.8. Let $\mathbf{A}=R$-modules, $R$ commutative, and let $A \in \mathbf{A}$. Let $G$ be a projective resolution of $R \in \mathbf{R}(\mathbf{T}, \mathbf{A})$. Then

$$
H_{n}(X \times Y, A)=H_{n}\left(G(X) \otimes_{R} G(Y) \otimes_{R} A\right)
$$

and

$$
H^{n}(X \times Y, A)=H^{n}\left(\operatorname{Hom}_{R}\left(G(X) \otimes_{R} G(Y), A\right)\right) .
$$

Proof. ( $\mathbf{T} \times \mathbf{T}, \mathbf{P} \times \mathbf{P}, \mathbf{E} \times \mathbf{E})$ is a base. Define a complex $G * G$ of functors in $\mathbf{R}(\mathbf{T} \times \mathbf{T}, \mathbf{A})$ by $(G * G)(X, Y)=G(X) \otimes G(Y)$. In the notation of the previous proof, $\left\langle P, A^{\prime}\right\rangle *\left\langle P^{\prime}, A^{\prime \prime}\right\rangle=\left\langle\left(P, P^{\prime}\right), A^{\prime} \otimes A^{\prime \prime}\right\rangle$, so that $G * G$ consists of projectives. $G(P)$ is a projective resolution of $R(P)$, and $\operatorname{Tor}_{n}\left(R(P), R\left(P^{\prime}\right)\right)=0, n \geq 1$. Hence $G(P) \otimes G\left(P^{\prime}\right)$ is acyclic over $R(P) \otimes R\left(P^{\prime}\right)$, so that $G * G$ is a projective resolution of $R * R$. Let $D(B)=B \otimes_{R} A$. Then, by Proposition 2.7,

$$
S_{n}(D(R * R))(X, Y)=H_{n}(G(X) \otimes G(Y) \otimes A) .
$$

Define $\Lambda: \mathbf{T} \times \mathbf{T} \rightarrow \mathbf{T}$ by $\Lambda(X, Y)=X \times Y$. Then $D(R * R)=A \Lambda$. (It suffices to check this on projectives.) Hence, since $\Lambda$ is a morphism of bases which preserves projectives, 


$$
S_{n}(D(R * R))(X, Y)=S_{n} A(X \times Y) .
$$

This is the first result. The second follows similarly.

Using the above corollary and theorem and the natural pairings, one obtains as usual the maps

$$
\begin{aligned}
& H^{n}(X, A) \otimes_{R} H_{m}(X \times X, B) \rightarrow H_{m-n}\left(X, A \otimes_{R} B\right), \\
& H^{n}(X, A) \otimes_{R} H^{m}(X, B) \rightarrow H^{n+m}\left(X \times X, A \otimes_{R} B\right)
\end{aligned}
$$

which define the cup and cap products.

$$
\text { Again, by Theorem 2.8, } H_{n}(X \times Y, A \otimes B)=H_{n}((G(X) \otimes A) \otimes(G(Y) \otimes B)),
$$
and

$$
\begin{aligned}
H^{n}(X \times Y, \operatorname{Hom}(A, C)) & =H^{n}(\operatorname{Hom}(G(X) \otimes G(Y) \otimes A, C)) \\
& =H^{n}(\operatorname{Hom}(G(X) \otimes A, \operatorname{Hom}(G(Y), C))) .
\end{aligned}
$$

When $R=Z$ and $\operatorname{Tor}_{1}(A, B)=0=\operatorname{Ext}^{1}(A, C)$, these equations and the above corollary combine as usual with the Künneth formulae [3, Chapter 5; $\$ 3$, Theorem 3 and Exercise C61 to yield split exact sequences

$$
\begin{aligned}
0 & \rightarrow \sum_{k+t=n} H_{k}(X, A) \otimes H_{t}(Y, B) \rightarrow H_{n}(X \times Y, A \otimes B) \\
& \rightarrow \sum_{k+t=n-1} \operatorname{Tor}_{1}\left(H_{k}(X, A), H_{t}(Y, B)\right) \rightarrow 0, \\
0 & \rightarrow \sum_{k+t=n-1} \operatorname{Ext}^{1}\left(H_{k}(X, A), H^{t}(Y, C)\right) \rightarrow H^{n}(X \times Y, \operatorname{Hom}(A, C)) \\
& \rightarrow \sum_{k+t=n} \operatorname{Hom}_{k}\left(H_{k}(X, A), H^{t}(Y, C)\right) \rightarrow 0,
\end{aligned}
$$

from which the Universal Coefficient Theorems follow as special cases.

Alternatively, these sequences can be derived directly. Let $\mathrm{A}$ be $R$-modules, $R$ commutative. From the commutative diagram

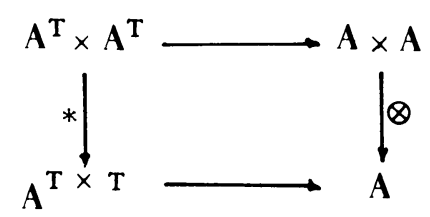

where the horizontal maps are evaluation at $(X, Y)$, we conclude $L_{n}(*)(F, G)(X, Y)$ $=\operatorname{Tor}_{n}^{R}(F(X), G(Y))$, where $L_{n}(*)$ is the $n$th left derived functor of $*$. Replacing $\mathbf{T}$ by $\mathbf{P}$, we obtain the same equation, when $X, Y \in \mathbf{P}$, for $*$ considered as a functor from $\mathbf{R}(\mathbf{T}) \times \mathbf{R}(\mathbf{T})$ to $\mathbf{R}(\mathbf{T} \times \mathbf{T}, \mathbf{A})$. Assume that $\operatorname{Tor}_{n}^{R}\left(F(P), G\left(P^{\prime}\right)\right)=0$ for $P, P^{\prime} \in \mathbf{P}, n \geq 1$. It follows that the left derived functors of the composition $\mathbf{R}(\mathbf{T}) \times \mathbf{R}(\mathbf{T}) \stackrel{*}{\rightarrow} \mathbf{R}(\mathbf{T} \times \mathbf{T}) \rightarrow \mathbf{A}^{\mathrm{T} \times \mathrm{T}}$ at $(F, G)$ are $S_{n}(F * G)$. Rewriting this composition as $\mathbf{R}(\mathrm{T}) \times \mathbf{R}(\mathrm{T}) \rightarrow \mathrm{A}^{\mathrm{T}} \times \mathrm{A}^{\mathrm{T}} \rightarrow \mathrm{A}^{\mathrm{T} \times \mathrm{T}}$, we obtain [1, Theorem 2] a spectral sequence 


$$
\sum_{k+l=q} \operatorname{Tor}_{p}^{R}\left(S_{k} F(X), S, G\left(Y^{\prime}\right)\right) \Rightarrow S_{n}(F * G)(X, Y)
$$

Introducing $\Lambda: \mathbf{T} \times \mathbf{T} \rightarrow \mathbf{T}$ as in the proof of Theorem 2.8, we see that $S_{n}(A * B)(X, Y)=S_{n}(A \otimes B)(X \times Y)$. Hence, if $R=Z$, the spectral sequence becomes a collection of exact sequences which generalize the first of those above. Similarly for the second sequence. They can be shown to split by induction on $n$. The argument is that of the proof of [1, Corollary to Theorem 1], making use of an exact sequence $0 \rightarrow F_{1} \rightarrow K \rightarrow F \rightarrow 0$ in $\mathbf{R}(\mathbf{T})$ with $K$ projective to reduce degree.

3. Simplicial homology. Excision. As in [0, Chapter II, 3.1], the spectral sequence associated to the derived functors of the composition $\mathbf{R} \rightarrow \mathbf{A}^{\mathrm{C}} \rightarrow \mathbf{A}$, where the first functor is inclusion and the second is $H_{0}(Y \rightarrow X,-)$, yields

Proposition 3.1. Let $(\mathbf{C}, \mathbf{P}, \mathbf{E})$ be any base, and let $F: \mathbf{C} \rightarrow \mathbf{A}$ be a right exact functor. Then, for any $Y \rightarrow X \in \mathbf{E}$, there is a spectral sequence

$$
H_{p}\left(Y \rightarrow X, s_{q} F\right) \Rightarrow s_{n} F(X) \text {. }
$$

(Note that the variance is reversed here, since we deal with covariant functors, while those in [0] are contravariant. We may assume that $\mathbf{C}$ is small; see Remark 2.3.)

Corollary. If $S_{q} F\left(Y^{n-q+1}\right)=0$ for all $1 \leq q \leq n$, where $Y^{m}$ denotes the $m$-fold fiber product $Y \times_{X} \cdots \times_{X} Y$, then $S_{n} F(X)=H_{n}(Y \rightarrow X, F)$.

Now let $\mathbf{C}$ be an arbitrary category. Then there is a category $\hat{\mathbf{C}}$, containing $\mathrm{C}$ as a full subcategory, with the following properties:

(1) Every directed system of objects in $\mathbf{C}$ has a $\underset{\lim }{\leftarrow}$ in $\hat{\mathbf{C}}$, and every object of $\hat{\mathbf{C}}$ arises in this fashion.

(2) If $\left\{X_{d}\right\}$ is a directed system of objects in $\mathbf{C}$, and if $Y \in|\mathbf{C}|$, then $\hat{\mathrm{C}}\left(\lim _{\leftarrow} X_{d}, Y\right) \stackrel{\lim }{\longrightarrow} \mathbf{C}\left(X_{d}, Y\right)$.

( $\hat{\mathbf{C}}$ is the dual of the full subcategory of functors from $\mathbf{C}$ to Sets whose objects are $\stackrel{\text { lim}}{\rightarrow}$ 's over directed systems of representable functors. $C$ is embedded in $\hat{\mathbf{C}}$ by $X \mapsto \vec{C}(X,-)$. Property (2) is an immediate consequence of the Yoneda lemma.)

Proposition 3.2. If $\mathbf{C}$ has fiber products, so does $\hat{\mathbf{C}}$, and the functor $\mathbf{C} \rightarrow \hat{\mathbf{C}}$ preserves fiber products.

Proof. By (2), $\hat{\mathbf{C}}\left(\lim _{\leftarrow} X_{d},-\right)$ carries fiber products in $\mathbf{C}$ to fiber products in Sets, which establishes the second fact. To see the first, let $\left\{X_{d}\right\}$ be a directed system of objects in $\mathbf{C}$, and $Y \in|\mathbf{C}|$. Given a morphism $\lim _{\leftarrow} X_{d} \rightarrow Y$, choose a representative $X_{d_{0}} \rightarrow Y$. Replacing $\{d\}$ by the cofinal subset $\left\{d \mid d \geq d_{0}\right\}$, we see 
that we can assume that our morphism is given by a coherent system of morphisms $X_{d} \rightarrow Y$. If $\lim _{\leftarrow} X^{\prime}{ }_{d^{\prime}} \rightarrow Y$ has the same property, then $\underset{\leftarrow}{\lim } X_{d}{ }{ }_{Y} \underset{\leftarrow}{\lim } X^{\prime}{ }_{d^{\prime}}$ exists because it is given by $\lim _{\leftarrow}\left(X_{d} \times_{Y} X_{d}^{\prime}\right)$, indexed over all pairs $\left(d, \overleftarrow{d^{\prime}}\right)$, with the obvious product ordering. Finally, if $\hat{X}$ and $\hat{X}^{\prime}$ are in $\hat{\mathbf{C}}$, and $\left\{Y_{b}\right\}$ is a directed system in C, $\hat{X} \times_{\lim _{\text {im }} Y_{b}} \hat{X}^{\prime}=\lim _{\longleftarrow}\left(\hat{X} \times_{Y_{b}} \hat{X}^{\prime}\right)$.

We now return to our topological base (T, P, E) (Definition 2.5). Let $\hat{\mathbf{E}}_{0}$ be all morphisms of the form $C^{\prime}+C^{\prime \prime}+\amalg C_{a} \rightarrow C+\amalg C_{a}$, where $C^{\prime}$ and $C^{\prime \prime}$ are subspaces of the cube $C$ which are homeomorphic to cubes, whose intersection is homeomorphic to a cube, and whose union is $C$; where $\left\{C_{a}\right\}$ is a finite set of cubes; and where the map is the canonical one. Let $\hat{\mathrm{E}}_{1}$ be all maps $\amalg Q_{i} \rightarrow \amalg P_{i}$ of finite coproducts, where each $P_{i}$ is a cube, each $Q_{i}$ is a finite disjoint union of subspaces of $P_{i}$ whose interiors cover, and the map is the canonical one; and where the intersection in $P_{i}$ of any subset of the subspaces comprising $Q_{i}$ is homeomorphic to a cube. Note that every morphism in $\hat{\mathbf{E}}_{1}$ can be written as a composition $f_{1} \cdots f_{n}$ of morphisms in $\hat{\mathbf{E}}_{0}$, with $f_{1} \cdots f_{k} \in \hat{\mathbf{E}}_{1}$ for every $k \leq n$. Let $C$ be a cube, and consider a sequence $\cdots \rightarrow C_{2} \rightarrow C_{1} \rightarrow C_{0}=C$, where $C_{i} \rightarrow C_{j} \in \hat{\mathbf{E}}_{1}$ whenever $i>j$, and where the diameters of the images in $C$ of the components of $C_{n}$ are less than a bound which goes to zero as $n$ approaches infinity. (Note that for any fixed $m$, the $m$-fold fiber products $\left\{C_{i} \times{ }_{C} \cdots \times{ }_{C} C_{i}\right\}$, being unions of intersections, again form such a sequence.) Clearly such sequences exist for any $C$. Let $\hat{\mathbf{P}}_{0} C|\hat{\mathbf{T}}|$ be all $\lim C_{i}$ over all such systems, as $C$ varies over all cubes. Given a set $\left\{\lim _{i} C_{a, i} \mid \alpha \in S\right\}$ of objects in $\hat{\mathbf{P}}_{0}$, let the set of maps $f: S \rightarrow Z^{+}$have the natural product order $\left(f_{1} \geq f_{2}\right.$ $f_{1}(\alpha) \geq f_{2}(\alpha)$ for every $\left.\alpha\right)$. Then the given set has a coproduct in $\hat{\mathbf{T}}$, given by

$$
\coprod_{a} \underset{i}{\lim } C_{a, i}=\underset{f: \underset{S \rightarrow Z^{+}}{\lim }}{\stackrel{\coprod}{S}} C_{a, f(a)}
$$

where the right-hand coproduct is taken in $\mathbf{T}$. Indeed, if $X \in|\mathbf{T}|$,

$$
\begin{aligned}
\hat{\mathbf{T}}\left(\lim _{f} \coprod_{a} C_{a, f(a)}, X\right) & =\underset{f}{\lim } \prod_{a} \mathbf{T}\left(C_{a, f(a)}, X\right)=\prod_{a} \underset{i}{\lim } \mathbf{T}\left(C_{a, i}, X\right) \\
& =\prod_{a} \mathbf{T}(\underbrace{\lim }_{i} C_{a, i}, X),
\end{aligned}
$$

which suffices to verify the above equation, since every $\hat{X}$ in $\hat{T}$ is a $\underset{\lim }{\leftarrow}$ of $X^{\prime}$ 's in $\mathbf{T}$. Let $\hat{\mathbf{P}}$ be all coproducts of objects in $\hat{\mathbf{P}}_{0}$, and let $\hat{\mathbf{P}}_{0} \Rightarrow \hat{\mathbf{E}}$ (as in Remark 2.4), so that $(\hat{\mathbf{T}}, \hat{\mathbf{P}}, \hat{\mathbf{E}})$ is a base.

Remark 3.3. A morphism $X^{\prime} \rightarrow X$ in $\mathbf{T}$ is in $\hat{\mathbf{E}}$ if, given any map from a cube to $X$, every point of the cube has a neighborhood such that the restriction of the map to the neighborhood factors through $X^{\prime}$.

Standing assumption. Throughout the remainder of this section we will assume that $\mathbf{A}$ has lim's over directed sets, and that $\underset{\leftarrow}{\lim }$ is exact in $\mathbf{A}$ over systems of 
epimorphisms indexed by the integers. This assumption is satisfied if $\mathbf{A}$ is a full category of modules or the dual thereof.

We wish now to extend the homology and cohomology functors to the category $\hat{\mathbf{T}}$. To this end, suppose that $F: \mathbf{T} \rightarrow \mathbf{A}$ is a right exact functor. If $\lim _{\leftarrow} P_{d} \in \hat{\mathbf{P}}$, let $\hat{F}\left(\lim _{\leftarrow} P_{d}\right)=\lim F\left(P_{d}\right)$. Define the value of $\hat{F}$ on morphisms between objects in $\hat{\mathbf{P}}$ in the natural fashion. Then $\hat{F}$ extends uniquely to a right exact functor defined on $\hat{\mathbf{T}}$ (see $\$ 1$ ). We have the category of pairs $\hat{\mathrm{T}}^{2}$, and $\hat{F}$ extends to a functor on $\hat{\mathrm{T}}^{2}$ (Definition 2.1). Then

Proposition 3.4. If $A \in|A|$, from the corresponding functor $A: \mathbf{T} \rightarrow \mathbf{A}$ define $\hat{A}: \hat{\mathbf{T}}^{2} \rightarrow \mathbf{A}$ as above. Then, if $(X, U) \in\left|\mathbf{T}^{2}\right|$,

$$
S_{n} \hat{A}(X, U)=S_{n} A(X, U) \quad \text { for every } n \geq 0 .
$$

Before proving this proposition, we see how the two main theorems of this chapter follow from it. We first need

Proposition 3.5. For any $A \in|\mathbf{A}|$, the functors $S_{n} A: \mathbf{T}^{2} \rightarrow \mathbf{A}$ preserve coproducts.

Proof. Let $f_{1}$ and $f_{2}$ be morphisms in $\mathbf{E}^{2}$ from projectives to two arbitrary objects in $\mathrm{T}^{2}$, and let $f_{1}+f_{2}$ be the corresponding morphism between coproducts. For $n \geq 1$, assume inductively that $S_{q} A$ preserves coproducts when $q<n$. Then the corresponding Čech complexes decompose to yield $H_{p}\left(f_{1}+f_{2}, S_{q} A\right)=$ $H_{p}\left(f_{1}, S_{q} A\right)+H_{p}\left(f_{2}, S_{q} A\right)(q<n)$. Since $f_{1}+f_{2}$ has a projective as domain, $H_{0}\left(f_{1}+f_{2}, S_{n} A\right)=0$. The result follows from the spectral sequence (Proposition 3.1).

Note. The usual singular complex for a coproduct breaks up into a direct sum of the complexes for the factors, so that, if we restricted ourselves to pairs whose morphism is injective, Proposition 3.5 would follow at once from Proposition 2.6. However, the aim of this paper is to use the methods of homological algebra to derive the properties of topological homology, and the argument for Proposition 2.6 used without proof the fact that the singular homology of a cube is trivial.

Theorem 3.6. Let $U_{1}$ and $U_{2}$ be subspaces of $X$ such that the canonical map $U_{1}+U_{2} \rightarrow X$ is in $\hat{\mathbf{E}}$. (Cf. Remark 3.3.) Let $V=U_{1} \cap U_{2}$. Let $V^{\prime} \rightarrow V$, $X \rightarrow X^{\prime}$ be continuous maps. Then there are exact Mayer-Vietoris sequences

$$
\begin{aligned}
\ldots \rightarrow S_{n} A\left(V, V^{\prime}\right) \rightarrow S_{n} A\left(U_{1}, V^{\prime}\right) & +S_{n} A\left(U_{2^{\prime}}, V^{\prime}\right) \\
& \rightarrow S_{n} A\left(X, V^{\prime}\right) \rightarrow S_{n-1} A\left(V, V^{\prime}\right) \rightarrow \cdots \\
\ldots \rightarrow S_{n} A\left(X^{\prime}, V\right) \rightarrow S_{n} A\left(X^{\prime}, U_{1}\right) & +S_{n} A\left(X^{\prime}, U_{2}\right) \\
& \rightarrow S_{n} A\left(X^{\prime}, X\right) \rightarrow S_{n-1} A\left(X^{\prime}, V\right) \rightarrow \cdots
\end{aligned}
$$


Proof. Let $G: \mathbf{T}^{2} \rightarrow \mathrm{A}$ preserve coproducts. Let $\theta$ be the canonical morphism $\left(U_{1}+U_{2}, V^{\prime}\right) \rightarrow\left(X, V^{\prime}\right)$. The Čech groups $H_{*}(\theta, G)$ are the homology groups of a complex with $n$th term the coproduct of all groups $G\left(U_{I}, V^{\prime}\right)$, where $I$ runs over all $(n+1)$-tuples composed of '1's and 2's and $U_{i_{0} \cdots i_{n}}=U_{i_{0}} \cap \cdots \cap U_{i_{n}}$. These homology groups are the same as those for the subcomplex with 0 th term $G\left(U_{1}, V^{\prime}\right)+G\left(U_{2}, V^{\prime}\right)$, 1st term $G\left(U_{12}, V^{\prime}\right)=G\left(V, V^{\prime}\right)$ and higher terms 0. (For instance, the map which sends $G\left(U_{I}, V^{\prime}\right)$ to $G\left(U_{1 I}, V^{\prime}\right)$ via the identity map if $I$ contains a 1 , and sends $G\left(U_{2} \ldots 2^{\prime} V^{\prime}\right)$ to 0 , is a homotopy between the identity and a map $\phi_{1}$; and, if $\phi_{2}$ is defined analogously by interchanging the roles of 1 and $2, \phi_{1} \phi_{2}$ is a homotopy inverse for the inclusion map.) Since the functors $S_{q} A$ preserve coproducts, it follows that $H_{p}\left(\theta, S_{q} A\right)=0, p \geq 2$, and that we have exact sequences

$$
\begin{aligned}
0 \rightarrow H_{1}\left(\theta, S_{q} A\right) & \rightarrow S_{q} A\left(V, V^{\prime}\right) \\
& \rightarrow S_{q} A\left(U_{1}, V^{\prime}\right)+S_{q} A\left(U_{2}, V^{\prime}\right) \rightarrow H_{0}\left(\theta, S_{q} A\right) \rightarrow 0
\end{aligned}
$$

On the other hand, applying Proposition 3.1 to the category $\hat{\mathbf{T}}^{2}$ with $Y \rightarrow X$ replaced by $\theta$ (which is in $\hat{\mathbf{E}}^{2}$ by assumption), and using Proposition 3.4, we obtain a spectral sequence

$$
H_{p}\left(\theta, S_{q} A\right) \Rightarrow S_{n} A\left(X, V^{\prime}\right)
$$

which, in the light of the above, is the first of the two desired Mayer-Vietoris sequences. The second follows similarly.

Remark. The excision axiom follows by taking $V^{\prime}=V, X^{\prime}=X$. Cf. [3, Chapter 4, Exercise I 1].

Theorem 3.7. Let $(X, U) \in \mathrm{T}^{2}$, and suppose that $\left\{X_{i}\right\}$ and $\left\{U_{i}\right\}$ are subspaces of $X, U$ respectively, and that each $U_{i}$ is contained in the inverse image of $X_{i}$. Let $\left(X^{\prime}, U^{\prime}\right)$ be the coproduct of the pairs $\left(X_{i}, U_{i}\right)$, and suppose that the canonical morphisms $X^{\prime} \rightarrow X$ and $U^{\prime} \rightarrow U$ are in $\hat{\mathbf{E}}^{2}$. (Cf. Remark 3.3.) Suppose further that, if $(Y, V)$ is the intersection of any subset of the set of pairs $\left(X_{i}, U_{i}\right)$, then $S_{k} A(Y, V)=0$ for all $1 \leq k \leq n$. Then $S_{n} A(X, U)=H_{n}\left(\left(X^{\prime}, U^{\prime}\right) \rightarrow(X, U), A\right)$, which is to say that the (co)bomology of $(X, U)$ with coefficients in $A$ can be computed using the simplicial complex associated to the nerve of the covering $\left\{X_{i}, U_{i}\right\}$. In particular, taking stars of vertices as the covering, it follows that singular and simplicial theory agree for simplicial complexes.

Proof. By hypothesis, $\left(X^{\prime}, U^{\prime}\right) \rightarrow(X, U) \in \hat{\mathbf{E}}^{2}$. The result follows from the corollary to Proposition 3.1 (applied in the category $\hat{\mathbf{T}}^{2}$ ), together with Proposition 3.4. (In verifying the hypothesis of the corollary, note that the fiber products which occur in the Čech complex are coproducts of pairs $(Y, V)$ which appear in the hypothesis of this theorem.) 
There remains to prove Proposition 3.4. We begin with

Lemma 3.8. $A$ is $\hat{\mathbf{E}}_{1}$-flask.

Proof. We claim first that $A$ is $\hat{\mathbf{E}}_{0}$-flask. In fact, since $A$ preserves coproducts, it suffices to show that $A$ is flask at morphisms in $\hat{\mathbf{E}}_{0}$ of the form $C^{\prime}+C^{\prime \prime} \rightarrow C$. As in the proof of Theorem 3.6, it is easy to see that $H_{n}\left(C^{\prime}+C^{\prime \prime} \rightarrow C, A\right)$ is the $n$th homology group of the complex $\cdots \rightarrow 0 \rightarrow \cdots \rightarrow$ $0 \rightarrow A\left(C^{\prime} \cap C^{\prime \prime}\right) \rightarrow A\left(C^{\prime}\right)+A\left(C^{\prime \prime}\right)=\cdots \rightarrow 0 \rightarrow \cdots \rightarrow 0 \rightarrow A \rightarrow A+A$, whence the claim follows. Next we will show by induction that, if $F: \mathbf{T} \rightarrow \mathbf{A}$ is any right exact functor which is $\hat{\mathbf{E}}_{0}$-flask, then it is $\hat{\mathbf{E}}_{1}$-flask, which will complete the proof. Let $P=\amalg C_{a}$ be a finite coproduct of cubes. For each $C_{\alpha}$, choose $\hat{C}_{a}=$ $\lim _{i} C_{a, i} \in \hat{\mathbf{P}}_{0}$ with $C_{a, 0}=C_{a}$. Let $P_{i}=\amalg_{a} C_{a, i}, \hat{\mathbf{P}}=\lim _{i} P_{i}=\amalg_{a} \hat{C}_{a}$. Then $\hat{P} \in \hat{\mathbf{P}}$, the canonical morphism $\hat{P} \rightarrow P$ is in $\hat{\mathbf{E}}$, and $\hat{P} \times{ }_{P} \hat{P}=\lim P_{i} \times{ }_{P} P_{i}=$ $\amalg_{a} \hat{C}_{a} \times_{C_{a}} \hat{C}_{a} \in \hat{\mathbf{P}}$. In the diagram

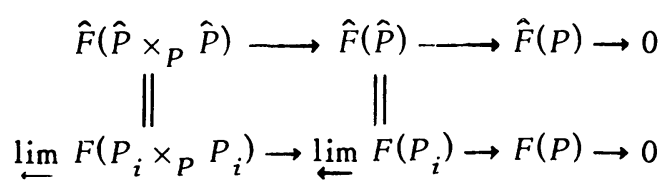

the top row is exact by definition. Since $\hat{\mathbf{E}}_{1}$ is generated under composition by $\hat{\mathbf{E}}_{0}, F$ carries $\hat{\mathbf{E}}_{1}$ to epimorphisms, so that the bottom row is exact by our exactness as sumption for $\lim$ in $A$. Hence $\hat{F}(P)=F(P)$.

Let $G \rightarrow \hat{F}$ be an epimorphism with $G$ a projective in $\mathbf{R}(\hat{\mathbf{T}})$. Let $K \in \mathbf{R}(\mathbf{T})$ be the kernel of the map from the restriction to $T$ of $G$ to the restriction to $T$ of $\hat{F}$ (i.e., $K$ is the right exact functor on $\mathbf{T}$ such that $0 \rightarrow K \rightarrow G \rightarrow F \rightarrow 0$ is exact on objects in $\mathbf{P}$ ). If $Q \rightarrow P \in \hat{\mathbf{E}}_{1}$, then the $m$-fold fiber product $Q^{m}=$ $Q \times{ }_{P} \cdots \times \times_{P} Q$ is a finite coproduct of cubes so that; by the above, $0 \rightarrow K\left(Q^{m}\right)$ $\rightarrow G\left(Q^{m}\right) \rightarrow F\left(Q^{m}\right) \rightarrow 0$ is exact. We obtain thus a short exact sequence of Cech complexes, whence a long exact sequence of $\check{C}$ ech homology groups. Since $G$ is $\hat{\mathbf{E}}$-flask, the latter becomes a sequence of isomorphisms

$$
H_{n+1}(Q \rightarrow P, F) \cong H_{n}(Q \rightarrow P, K) \quad(n \geq 1)
$$

and an exact sequence

$$
0 \rightarrow H_{1}(Q \rightarrow P, F) \rightarrow H_{0}(Q \rightarrow P, K) \rightarrow H_{0}(Q \rightarrow P, G) \rightarrow H_{0}(Q \rightarrow P, F) \rightarrow 0 .
$$

In particular, taking $Q \rightarrow P$ to be in $\hat{\mathbf{E}}_{0}$, we see that $K$ is $\hat{\mathbf{E}}_{0}$-flask. Since $\hat{\mathbf{E}}_{1}$ is generated under composition by $\hat{\mathbf{E}}_{0}$, it follows that $K$ as well as $F$ carries $\hat{\mathbf{E}}_{1}$ to epimorphisms, and then, by Remark 1.2 , that $F$ and $K$ respect all morphisms in $\hat{\mathbf{E}}_{1}$. Taking $Q \rightarrow P \in \hat{\mathbf{E}}_{1}$, we then see from the last sequence above that $H_{1}(Q \rightarrow P, F)=0$.

We can now replace $F$ by $K$ and proceed by induction, thus completing the proof. 
Lemma 3.9. If $P \in \mathbf{P}, \hat{A}(P)=A(P)$ and $S_{n} \hat{A}(P)=0$ for all $n \geq 1$.

Proof. $P$ is a coproduct of cubes: $P=\amalg C_{a}$. For each $\alpha$, choose $\hat{C}_{a}=$ $\lim C_{a, i} \in \hat{\mathbf{P}}_{0}$ with $C_{a, 0}=C_{a}$. Let $\hat{P}=\amalg \hat{C}_{a}$, and let $\hat{P} \rightarrow P$ be the canonical morphism. Denote the $m$-fold fiber products $\hat{C}_{a} \times_{C_{a}} \cdots \times_{C_{a}} \hat{C}_{a}, \hat{P} \times_{P} \cdots \times_{P} \hat{P}$ and $C_{a, i} \times{ }_{C_{a}} \cdots \times{ }_{C_{a}} C_{a, i}$ by $\hat{C}^{m}, \hat{P}^{m}$ and $C_{a, i}^{m}$ respectively. Then $\hat{C}^{m}=\lim C_{a, i}^{m}$ is again in $\hat{\mathbf{P}}_{0}$. As we remarked in the previous proof, it is easy to see that $\hat{P} \rightarrow P \in \hat{\mathbf{E}}$ if the coproducts involved are finite. In the infinite case, since any morphism from an object in $\hat{\mathbf{P}}_{0}$ to $P$ factors through the coproduct of a finite number of its components, the fact that $\hat{P} \rightarrow P \in \hat{\mathbf{E}}$ follows from the finite case. Now (cf. the proof of Proposition 3.2)

$$
\begin{aligned}
& \hat{P} \times_{P} \hat{P}=\lim _{\left(f_{1}, f_{2}\right)} \coprod_{a} c_{a, f_{1}(a)} \times{ }_{\mathbf{U} C_{a}} \coprod c_{a_{1} f_{2}(a)} \\
& =\underset{f_{1}, f_{2}}{\lim _{a}} \coprod_{a} C_{a_{0} f_{1}(a)} \times C_{a} C_{a_{0} f_{2}(a)}=\underbrace{\lim }_{f} \coprod_{a} C_{a_{0} f(a)} \times C_{a} C_{a_{0} f(a)} \\
& =\coprod_{a} \underset{i}{\lim } C_{a, i} \times C_{a} C_{a, i}=\coprod_{a} \hat{C}_{a} \times C_{a} \hat{C}_{a},
\end{aligned}
$$

and, similarly,

$$
\hat{P}^{m}=\underset{f}{\lim } \coprod_{a} C_{a, f(a)}^{m}=\coprod_{a} \hat{C}_{a}^{m}
$$

In particular, $\hat{P}^{m} \in \hat{\mathbf{P}}$. Hence, by the corollary to Proposition 3.1,

$$
S_{n} \hat{A}(P)=H_{n}(\hat{P} \rightarrow P, \hat{A}) \quad(n \geq 0)
$$

$H_{n}(\hat{P} \rightarrow P, \hat{A})$ is the $n$th homology group of the complex with $n$th group

$$
\hat{A}\left(\hat{P}^{m}\right)=\hat{A}\left(\underset{\leftarrow}{\lim _{f}} \coprod_{a} C_{a, f(a)}^{m}\right)=\underset{f}{\lim } \coprod_{a} A\left(C_{a, f(a)}^{m}\right)=\coprod_{a} \underset{i}{\lim } A\left(C_{a, i}^{m}\right) .
$$

Our exactness assumptions about coproducts and $\lim$ in $\mathbf{A}$ allow us to conclude that

$$
H_{n}(\hat{P} \rightarrow P, \hat{A})=\coprod_{a} \underset{i}{\lim } H_{n}\left(C_{a, i} \rightarrow C_{a^{\prime}} A\right),
$$

and the desired result follows from the previous lemma.

Proof of Proposition 3.4. The inclusion functor $\mathrm{T}^{2} \rightarrow \hat{\mathrm{T}}^{2}$ preserves fiber products and carries $\mathbf{E}^{2}$ to $\hat{\mathbf{E}}^{2}$. The first part of Lemma 3.9 tells us that the restriction of $\hat{A}$ to $\mathrm{T}^{2}$ is $A$ (since both these functors are right exact and agree on projectives). From the second part of Lemma 3.9 and exactness (2.2) we see that $S_{n} \hat{A}\left(P, P^{\prime}\right)=0$ for $n \geq 1,\left(P, P^{\prime}\right) \in \mathbf{P}^{2}$. The proposition then follows from the Comparison Theorem (Theorem 3.1). 


\section{BIBLIOG RA PHY}

0. M. Artin, Grothendieck topologies, Harvard University, Cambridge, Mass., 1962 (mimeographed notes).

1. G. S. Rinehart, Note on the homology of a fiber product of groups, Proc. Amer. Math. Soc. 24 (1970), 548-552. MR $41 \# 1838$.

2. G. S. Rinehart, Satellites and cohomology, J. Algebra 12 (1969), 295-329; Errata, ibid. 14 (1970), 125-126. MR 39 \#6953; MR 40 \#1455.

3. E. H. Spanier, Algebraic topology, McGraw-Hill, New York, 1966. MR 35 \#1007.

DEPARTMENT OF MATHEMATICS, CORNELL UNIVERSITY, ITHACA, NEW YORK 14850 